\title{
Maintaining a stochastic neuronal cell fate decision
}

\author{
Daniel Vasiliauskas, Robert Johnston, and Claude Desplan ${ }^{1}$ \\ Department of Biology, New York University, New York, New York 10003, USA
}

\begin{abstract}
Sensory systems generally contain a number of neuronal subtypes that express distinct sensory receptor proteins. This diversity is generated through deterministic and stochastic cell fate choices, while maintaining the subtype often requires a distinct mechanism. In a study published in the February 1, 2009, issue of Genes \& Development, Lesch and colleagues (pp. 345-358) describe a new transcription factor, NSY-7, that acts to stabilize a stochastic subtype choice in AWC chemosensory neurons in Caenorhabditis elegans.
\end{abstract}

Much work in developmental neurobiology has focused on understanding how neuronal diversity is generated. Less, however, has been done to investigate how this diversity is maintained. Sensory systems provide excellent opportunities to study both of these issues. For instance, olfactory and visual systems contain a large variety of neuronal subtypes that are defined by the sensory receptor proteins they express. Expression of sensory receptor proteins is coordinated with other functional attributes such as cell morphology, axonal projection pattern, connectivity, and expression of signal transduction pathway genes. Sometimes, communication between different neurons is essential for the coordination of specific sensory identities. Thus, a wealth of information has been gathered through the investigation of how decisions are made to express one or a particular constellation of sensory receptors out of a number of alternatives.

\section{Determinate versus stochastic choices}

Although plasticity clearly requires input from the environment, most decisions in the central nervous system are determinate. Lineage information and inductive signaling/guidance cues direct a neuron toward a given fate and specific connectivity. However, sensory systems sometimes rely on stochastic decisions to generate the

[Keywords: Homeodomain; olfactory development; transcriptional maintenance; transcriptional repression; activity-dependent gene expression; cGMP-dependent protein kinase]

${ }^{1}$ Corresponding author.

E-MAIL cd38@nyu.edu; FAX (212) 995-4710.

Article is online at http://www.genesdev.org/cgi/doi/10.1101/gad.1780509. diversity of sensory neurons that can detect the multitude of sensory stimuli present in the environment. In the olfactory system in mice, for example, each neuron makes a stochastic decision to express one of over a thousand olfactory receptor genes, and to exclude expression of all others (for review, see Johnston and Desplan 2008). Stochastic decisions can happen in two general ways: They can be intrinsic to the cell or they can involve communication between neighboring cells (for review, see Losick and Desplan 2008). This coordination of randomized cell fates is particularly important when there are a limited number of cells and every alternative subtype must be represented.

Little is known about how sensory cells make intrinsic stochastic subtype fate choices, and this question represents one of the most challenging problems in neurobiology. In fact, the study of how any cell type makes such decisions is still a young and blossoming field. Major advances in our understanding of the role of noisy gene expression to generate stable cell fates have occurred primarily in bacteria. Very elegant experiments in Bacillus subtilis have highlighted the basic principles of this process: noisy gene expression, bistable choice, and robustness/maintenance. In the case of $B$. subtillis competence, noise in the expression of a transcriptional regulator, ComK, is utilized to generate one of two decisions, to enter a program of competence to accept foreign DNA, or not. When ComK levels reach a threshold, an autoregulatory bistable loop ensures that the bacterium commits to one fate rather than oscillating between fates. Each bacterium makes its choice independently from others (Maamar and Dubnau 2005; Suel et al. 2006; Maamar et al. 2007).

In the case of coordinate stochastic decisions, communication between two or more cells is an integral part of the choice mechanism. Cells compete with each other ("lateral inhibition") to ensure that the two alternatives are chosen at the appropriate frequency. A classic example of this type of mechanism is the generation of small sensory bristles in the peripheral nervous system of the fruitfly. Random fluctuations in the Delta-Notch-mediated signaling among a group of cells leads to a "winner" microchaete precursor that prevents its neighbors from making the same choice (for review, see Simpson 1997). If this cell is eliminated experimentally, the inhibitory signal is no longer present, the competition can start again, and 
another cell can become the neuroblast. Another example of a nonintrinsic stochastic decision, discussed below, is the subtype choice by AWC sensory neurons in Caenorhabditis elegans.

\section{Maintenance}

Neurons live a long time, often the lifetime of the individual. If the identity of a sensory receptor were to switch midlife, without the corresponding change in other attributes of the neuron such as connectivity, sensory confusion would result. For example, in the Drosophila eye, there is no turnover of photoreceptor cells. The color photoreceptors acquire their subtype identity by expressing a specific Rhodopsin (Mikeladze-Dvali et al. 2005; Wernet et al. 2006; Mazzoni et al. 2008). The R8 photoreceptors that express blue- or green-sensitive Rhodopsins project to more superficial layers of the medulla in the optic lobe, while UV-sensitive R7 photoreceptors project to deeper layers of the medulla (Fischbach and Dittrich 1989; Morante and Desplan 2004). Hence, if a photoreceptor were to switch Rhodopsin expression mid-life without a corresponding change in the projection pattern, it would convey misleading information to the wrong part of the brain (Cook et al. 2003; Wernet and Desplan 2004). Thus, it is essential that specific sensory protein expression is maintained throughout the life of the sensory neurons, which often means throughout the life of the animal. And while it is possible that the mechanisms and genes that act to set up the subtype identity might also function to maintain it, maintenance is likely to require dedicated mechanisms.

Sensory neurons of different subtypes can be very similar to each other: They may share common developmental history and gene expression batteries until late differentiation events determine subtype-specific sensory receptor expression. Thus, it is reasonable to expect that the probability of inappropriate subtype switching, if not actively repressed, could be quite high. This is well illustrated by the mouse olfactory system. Each of the olfactory sensory neurons expresses one allele of over a thousand olfactory receptor genes (Chess et al. 1992; Malnic et al. 1999). A fair proportion of these genes are pseudogenes encoding nonfunctional olfactory receptor proteins, whose expression would render the cell useless. If a cell chooses to express a functional olfactory receptor, it is very unlikely to switch to another gene. However, if the initial choice leads to expression of a nonfunctional olfactory receptor, the probability of switching increases significantly. Thus, the expression of a functional receptor acts not only to maintain the subtype of the cell, but also participates in its establishment (Serizawa et al. 2000; Lewcock and Reed 2004; Shykind et al. 2004).

\section{Lateral asymmetry in worm olfactory AWC neurons}

The study of the worm, C. elegans, has provided numerous insights into the lineage and signaling events required for cell fate determination. Consisting of $\sim 1000$ cells, the worm relies predominantly on the conveyance and in- terpretation of lineage information during development to yield a nearly perfectly reproducible pattern of cell fates. With few exceptions, the cell lineage of the worm can be traced from the point of fertilization through primarily anterior/posterior division patterns to specific cell types (Sulston and Horvitz 1977).

One exception to the determinant lineage of the worm is the case of the AWC class of chemosensory neurons that detect volatile odors (Bargmann et al. 1993). This bilateral pair of neurons displays general cell fate characteristics (specific gene expression, cell morphology) derived from lineage information. However, they deviate from one another in the expression of specific chemoreceptor proteins, yielding two subtypes, $\mathrm{AWC}^{\mathrm{ON}}$ and AWC ${ }^{\mathrm{OFF}}$ : $\mathrm{AWC}^{\mathrm{ON}}$ fate is defined by expression of the str-2 gene, while $\mathrm{AWC}^{\mathrm{OFF}}$ cell fate is defined by expression of srsx-3 (Troemel et al. 1999; Bauer Huang et al. 2007). It is thought that these receptor-like proteins endow the AWC cells with distinct chemosensory sensitivities. Interestingly, in wild-type animals, one AWC cell is always $\mathrm{AWC}^{\mathrm{ON}}$ while the other is $\mathrm{AWC}{ }^{\mathrm{OFF}}$, but the decision whether the left or the right cell becomes $\mathrm{AWC}^{\mathrm{ON}}$ is stochastic. Over the last 15 years, the Bargmann laboratory has applied genetic, cellular ablation, and electrophysiological approaches to provide a thorough description of the communication between AWC right and left neurons that leads to the mutually exclusive and randomly selected $\mathrm{AWC}^{\mathrm{ON}}$ and $\mathrm{AWC} \mathrm{OFF}^{\mathrm{O}}$ fates.

\section{Lateral signaling between AWC cells determines subtype fate}

The first insight into the cell fate decision and coordination mechanism employed by the AWC neurons was provided by laser ablation experiments (Troemel et al. 1999). Ablation of one AWC neuron precursor always leads to $\mathrm{AWC}^{\mathrm{OFF}}$ fate in the remaining neuron. This observation suggested a role for cellular communication between the AWC neurons for subtype specification. To explore the mechanisms involved in this process in more detail, the Bargmann laboratory (Troemel et al. 1999) performed a thorough genetic screen for mutations that disrupt asymmetric expression of str-2, resulting in either a phenotype with two $\mathrm{AWC}^{\mathrm{ON}}$ or two AWC ${ }^{\mathrm{OFF}}$ cells. Cloning and characterization of mutants isolated from the genetic screens allowed them to uncover an elaborate electrical signaling-based mechanism by which AWC neurons use lateral inhibition to ensure that only one cell becomes $\mathrm{AWC}^{\mathrm{ON}}$ and the other $\mathrm{AWC}^{\mathrm{OFF}}$. Initially, a membrane potential flows through a network of neuroblasts that include AWC precursors. It appears that stochastic differences in potential determine directionality of this flux from AWC left to AWC right or vice versa. The claudin, NSY-4, and the innexin-type gap junction protein, NSY-5, are key components of this mechanism (Vanhoven et al. 2006; Chuang et al. 2007). High activity of these proteins leads to changes in the cell's membrane potential that induce $\mathrm{AWC}^{\mathrm{ON}}$ fate by repressing $\mathrm{Ca}^{2+}$ influx through voltage-gated calcium channels (UNC-2/ UNC-36). In the absence of $\mathrm{Ca}^{2+}$ influx, CaMKII is inactive, 
preventing stimulation of a downstream MAP kinase cascade (NSY-1, SEK-1, TIR-1) and leading to activation of the $\mathrm{AWC}^{\mathrm{ON}}$ fate and repression of the AWC ${ }^{\mathrm{OFF}}$ fate. In the opposite cell, low activity of NSY-4 and NSY-5 allows $\mathrm{Ca}^{2+}$ influx activating CaMKII and MAP kinase cascades, yielding activation of the $\mathrm{AWC}{ }^{\mathrm{OFF}}$ fate and repression of the AWC $^{\text {ON }}$ fate (Sagasti et al. 2001; Tanaka-Hino et al. 2002; Chuang and Bargmann 2005; Bauer Huang et al. 2007).

To ensure randomization of lateral inhibition, the predetermined AWC cells must be equivalent in their ability to take on $\mathrm{AWC}^{\mathrm{ON}}$ or $\mathrm{AWC}^{\mathrm{OFF}}$ fate. The simplest hypothesis would be that each cell expresses the same set of molecules with the same capabilities of inducing cell fate. However, somewhat surprisingly, during their characterization of the key players, nsy-4 and nsy-5, the Bargmann laboratory (Chuang et al. 2007) found that the AWC precursor cells on the left and on the right have unique predetermined properties. Initially, the left AWC cell is inherently more responsive to nsy-4 activity, whereas the right AWC cell is more responsive to nsy-5 activity. Since NSY-4 and NSY-5 both induce AWC ${ }^{\mathrm{ON}}$ fate, both cells apparently pursue $\mathrm{AWC}^{\mathrm{ON}}$ fate, although in different ways. However, slight activity differences generate a membrane potential in one direction, inducing high NSY-4 and NSY-5 activity in one cell compared with the other, yielding $\mathrm{AWC}^{\mathrm{ON}}$ fate.

\section{Establishment versus maintenance of the fate decision}

Characterization of the numerous mutants identified in the genetic screens has led to several interesting insights into mechanisms by which the two AWC subtypes are specified. There appear to be two distinct steps: establishment and maintenance (Troemel et al. 1999). When genes involved in establishment such as nsy-4 and nsy-5 are mutated, $\mathrm{AWC}^{\mathrm{ON}}$ fate is never specified and str-2 is never expressed. In mutants defective for maintenance, str-2 is asymmetrically expressed in larvae, indicating that the $\mathrm{AWC}^{\mathrm{ON}}$ cell fate has been chosen, but this expression is not sustained (Troemel et al. 1999). These genes encode chemosensory signal transduction molecules such as guanylyl cyclases (ODR-1 and DAF-11), cGMP-gated channel subunits (TAX-2, TAX-4) (Troemel et al. 1999), a cGMP-dependent kinase homolog (EGL-4) (Lesch et al. 2009), and olfactory G $\alpha$ proteins (ODR-3, GPA-2) (Lans et al. 2004; Lans and Hoeijmakers 2006).

In a study in the February 1, 2009, issue of Genes \& Development, the Bargmann group (Lesch et al., 2009) turned its attention to the transition between the point when the AWC cell chooses its subtype and the establishment of stable expression of str-2. They describe a mutation in a gene identified in their screen, nsy-7 (Vanhoven et al. 2006), which has a "maintenance" phenotype but exhibits significant differences from the maintenance mutants involved in olfactory transduction.

In order to understand the transition between the establishment and maintenance of the $\mathrm{AWC}^{\mathrm{ON}}$ identity, Lesch et al. (2009) carefully examined the temporal dynamics of expression during larval development of the two markers of terminal differentiation of the
$\mathrm{AWC}^{\mathrm{ON}}$ and $\mathrm{AWC}^{\mathrm{OFF}}$ fates, str-2 and srsx-3. For this purpose, Lesch et al. (2009) generated a new tool, a transgenic strain carrying a green reporter for srs $x-3$ expression (srsx-3::GFP), which marks the AWC ${ }^{\mathrm{OFF}}$ subtype, and a red reporter for str-2 expression (str-2:: dsRED), which marks the AWC ${ }^{\mathrm{ON}}$ subtype (Bauer Huang et al. 2007). This allowed a direct comparison and correlation of the two markers in the $\mathrm{AWC}^{\mathrm{ON}}$ and $\mathrm{AWC} \mathrm{OFF}^{\mathrm{O}}$ cells. In the newly hatched larvae, srsx-3::GFP is expressed in both AWC cells, but it is already five times higher in the presumptive $\mathrm{AWC}^{\mathrm{OFF}}$ cell. During the L1 larval stage, srsx-3::GFP continues to increase in both cells, but subsequently drops in the $\mathrm{AWC}^{\mathrm{ON}}$ cell while reaching maximum adult levels in the $\mathrm{AWC}^{\mathrm{OFF}}$ cell. Asymmetric expression of str- $2:: d s R E D$ appears in a single AWC cell at mid-L1, well after the AWC ${ }^{\text {OFF }}$ state has been established, as marked by strong $\operatorname{srsx}-3:$ : GFP expression in one of the two neurons. str-2:: dSRED-positive AWC is invariably the one with the lower levels of $s r s x-3:: G F P$. str- $2:: d s R E D$ levels then continue to increase in the $\mathrm{AWC}^{\mathrm{ON}}$ cell. Therefore, there is a significant difference in the timing of appearance of the $\mathrm{AWC}^{\mathrm{ON}}$ and $\mathrm{AWC}{ }^{\mathrm{OFF}}$ markers, which suggests that the decision to turn off the AWC ${ }^{\mathrm{OFF}}$ fate precedes that of gaining str-2 expression.

What allows AWC cells to firmly commit to their fate? Are there genes required for the transition between subtype choice and stable identity? It appears that nsy-7, fulfills several of the requirements to play this role. This mutant fails to express the str-2 reporter in either AWC adult neuron. However, the nsy-7 phenotype differs significantly from the phenotypes of mutations in genes, such as nsy-5, that participate in the AWC subtype identity decision: While str-2 is never induced in nsy-5 mutants, it is expressed in nsy-7 mutants at larval stages. This observation suggested that the decision to become $\mathrm{AWC}^{\mathrm{ON}}$ or $\mathrm{AWC} \mathrm{OFF}^{\mathrm{O}}$ is, in fact, made in nsy-7 mutants. Careful characterization of the dynamics of appearance of the terminal differentiation reporters of $\mathrm{AWC}^{\mathrm{ON}}$ and AWC ${ }^{\mathrm{OFF}}$ cells in nsy-7 mutants showed that srsx-3::GFP is expressed in both AWC cells with a time course similar to its expression in wild-type AWC ${ }^{\mathrm{OFF}}$ cells. While the levels of srsx-3::GFP expression between the two AWC cells of individual mutant animals generally differ, they are within the normal range for the $\mathrm{AWC}^{\mathrm{OFF}}$ cells. This suggests that no decision is made to turn off the AWC ${ }^{\mathrm{OFF}}$ state in nsy-7 mutants. However, str-2::dsRED does appear, and it does so in only one of the two AWC cells by the end of L1 larval stage, although at levels significantly lower than for wild-type $\mathrm{AWC}^{\mathrm{ON}}$ cells. Importantly, this expression does not mirror the difference in srsx-3::GFP levels between the two AWC cells; i.e., it does not correlate with the cell with lower srsx-3 expression. Subsequently, the expression of $s t r-2:: d s R E D$ drops, although a proportion of adults still retain it at low levels. Since the fate of the two AWC subtypes is fixed by the L1/ L2 transition in the wild type, Lesch et al. (2009) conclude that the choice of subtype is not affected in nsy-7 mutants, but that the subtype identity fails to be maintained. 


\section{nsy-7 is the first marker of $\mathrm{AWC}^{\mathrm{ON}}$ identity}

Lesch et al. (2009) cloned the nsy-7 gene and identified a DNA fragment upstream of the gene that can direct reporter gene expression (nsy-7::GFP). In adults, nsy-7:: GFP is expressed in $\mathrm{AWC}^{\mathrm{ON}}$ cells and not in $\mathrm{AWC}^{\mathrm{OFF}}$ cells. It is first observed in embryonic stages, at the time when AWC asymmetry is initiated; i.e., well before the final $\mathrm{AWC}^{\mathrm{ON}}$ differentiation marker str-2::dsRED is turned on. Already at this stage, most embryos express nsy-7::GFP in one AWC cell only.

Therefore, nsy-7 is the earliest asymmetrically expressed marker correlated with subtype specification of AWC cells. Lesch et al. (2009) thus asked whether it is regulated by the genes that act to set up the $\mathrm{AWC}^{\mathrm{ON}}$ and $\mathrm{AWC}^{\mathrm{OFF}}$ cell fates. Mutations in the nsy-5, result in a 2 -AWC ${ }^{\text {OFF }}$ phenotype (Chuang et al. 2007) and also lead to loss of nsy-7 expression. Conversely, mutations in $n s y-1$ result in a 2 -AWC ${ }^{\mathrm{ON}}$ phenotype (Troemel et al. 1999) and lead to expansion of nsy-7::GFP into both AWC cells. Interestingly, the double mutant combinations of nsy-1 (or unc-2 or unc-36) (2-AWC ${ }^{\mathrm{ON}}$ ) with nsy-7 result in the nsy-7 phenotype (2-AWC $\left.{ }^{\mathrm{OFF}}\right)$, arguing that $n s y-7$ acts downstream from these genes. Thus, nsy-7 is both transcriptionally regulated by the signaling pathway that specifies the two AWC subtypes, and it is necessary for this pathway to produce AWC ${ }^{\mathrm{ON}}$ cells. nsy-7 is, in fact, more than simply required for the maintenance of $\mathrm{AWC}^{\mathrm{ON}}$ fate: It is also sufficient for this fate as forced expression of nsy-7 in AWC cells turns them both into the AWC ${ }^{\mathrm{ON}}$ subtype (see below).

How does nsy-7 function relate to other genes that are also involved in the maintenance of str-2? In contrast to the nsy-7 phenotype, in sensory transduction mutants odr-1 and egl-4 mutants, both str-2::dsRED and srsx$3:: G F P$ are expressed at L1, but lost in the adult. Thus, continuous expression of str-2 and srsx-3 requires an active sensory signal transduction pathway that acts in both $\mathrm{AWC}^{\mathrm{ON}}$ and $\mathrm{AWC}{ }^{\mathrm{OFF}}$ cells. The same phenotype is observed when these mutations are placed in a nsy-7 mutant background. Thus, nsy-7, at least with respect to maintenance of srsx-3 expression, acts upstream of the chemosensory signal transduction pathway. It is there- fore possible that the cells keep their subtype identity in odr-1 and egl-4 mutants but fail to maintain expression of their final differentiation markers. It will be important to figure out if the specification is maintained in these mutants by determining whether nsy-7::GFP is still expressed asymmetrically or not. In the case of nsy-7 mutants, the situation is quite different: The AWC ${ }^{\mathrm{ON}}$ subtype is initially established but appears to revert later on, with the $\mathrm{AWC}^{\mathrm{OFF}}$ fate replacing the $\mathrm{AWC}^{\mathrm{ON}}$.

\section{nsy-7 acts as a transcription factor to maintain the AWC $^{\text {ON }}$ identity}

NSY-7 protein has a domain similar to a homeodomain, localizes to the nucleus, and binds a specific DNA sequence, all consistent with a direct role as a transcription factor. Using in vitro approaches, Lesch et al. (2009) identified the DNA sequence preferred by NSY-7. This turned out to be very fruitful: Lesch et al. found this sequence upstream of the srsx-3 gene and showed that it is part of a larger compound site. One-half of this site is necessary to activate expression of a reporter, while the other bases are recognized by NSY-7 and mediate its repression in $\mathrm{AWC}^{\mathrm{ON}}$ cells. Thus, NSY-7 appears to be a transcriptional repressor that directly down-regulates srs $x$-3. In contrast, induction of str-2 occurs considerably later, suggesting that either NSY-7 regulates str-2 together with a cofactor that only becomes available mid-L1 or, more likely, that nsy-7 up-regulates str-2 expression indirectly, perhaps by repressing a repressor.

\section{Perspectives}

The work on the specification of AWC neurons has provided many new insights into mechanisms of stochastic cell fate decisions, including how cells make decisions in concert with each other. This new study by Lesch et al. (2009) adds one more dimension by emphasizing the need for maintenance of the neuronal subtype by nsy-7 and at a different level, maintenance of sensory receptor expression by the sensory transduction pathway.

A number of important questions remain concerning nsy-7. The consistently asymmetric expression of str-2 at
Figure 1. A model for the role of NSY-7 in establishment and maintenance of stable AWC chemosensory neuron subtypes. See the text for details.
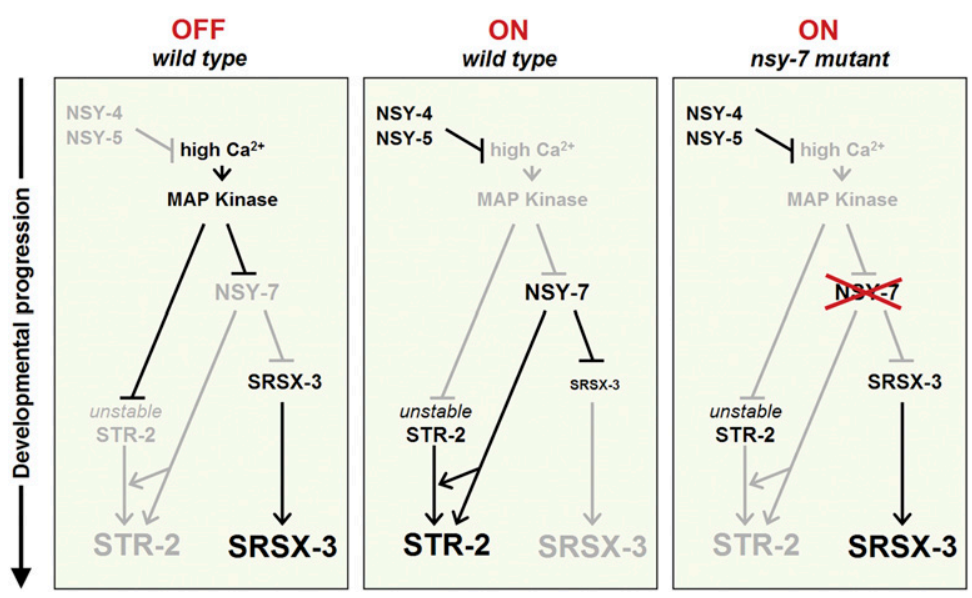
the L1/L2 transition during larval development in nsy-7 mutant animals indicates progression toward the AWC ${ }^{\mathrm{ON}}$ fate. At this point, cell ablation experiments in wild-type animals indicate that cell-cell communication no longer plays a role in AWC fate decision. However, the low levels of str-2 expression in mutant cells suggest that the differentiation is not complete. So, what is the exact status of the str-2-expressing cells in wild type and nsy-7 mutants? In mutant cells, srsx-3 expression does not appear to be down-regulated. Therefore, it is possible that the $\mathrm{AWC}^{\mathrm{ON}}$ fate is only weakly specified, and the absence of nsy-7 function leads to the inability to repress the AWC ${ }^{\mathrm{OFF}}$ state represented by $s \mathrm{~S}_{\mathrm{S} X}-3$ expression. The study by Lesch et al. (2009) provides a molecular explanation to the lack of down-regulation of srsx-3: NSY-7 appears to directly repress $s r s x-3$ expression. Since nsy-7::GFP itself is the earliest marker of AWC asymmetry that is downstream from the subtype choice machinery, it would be very interesting to know whether it is asymmetrically expressed early in nsy-7 mutants. This would constitute powerful further evidence that the AWC subtype decision has been made.

The discovery of $n s y-7$ as a fate stabilizing/maintenance gene invites a number of questions regarding its function. Is nsy-7 required initially to establish a stable state, or does it act continuously to maintain it, or both (Fig. 1)? The early asymmetric expression and early function of nsy-7 suggests that it acts in the initial stabilization of a chosen fate $\left(\mathrm{AWC}^{\mathrm{ON}}\right)$. Furthermore, the asymmetric expression pattern as well as the fact that nsy-7 is both necessary and sufficient to impart $\mathrm{AWC}^{\mathrm{ON}}$ identity suggest that it does not simply stabilize the gene expression that is already present, but in fact takes over the control of the subtype (Fig. 1). It is less clear whether nsy-7 is continuously required to maintain the $\mathrm{AWC}^{\mathrm{ON}}$ fate. To ascertain this, temperature-sensitive or otherwise conditional mutants will be required.

The next question is to understand how the expression of nsy-7 is controlled. Its asymmetric expression is clearly established as a consequence of the choice that involves producing unequal $\mathrm{Ca}^{2+}$ concentrations in the two AWC cells. Is nsy-7 necessary for the communication between the two cells, which could be suggested by the independent expression of str-2 and low srsx-3 in the mutant? Once nsy-7 expression is established, does it need to be maintained and, if so, does it involve positive autoregulation in $\mathrm{AWC}^{\mathrm{ON}}$ cells?

Once str-2 expression is established in $\mathrm{AWC}^{\mathrm{ON}}$ neurons, it is maintained. However, there is one exception: In the Dauer stage, $\mathrm{AWC}^{\mathrm{ON}}$ neurons down-regulate str-2, and presumably re-express it when the Dauer returns to an active lifestyle (Peckol et al. 2001). It would be interesting to ask what happens to nsy-7 expression as the animal enter and exits the Dauer state. Does nsy-7 lead the way?

\section{Conclusion}

What general lessons can nsy-7 teach us about sensory systems? Although nsy-7 does not seem to have homologs in other model organisms, the principle that a specific transcription factor can act to stabilize/maintain a corresponding sensory subtype, while repressing alternative cell fates could hold true for other sensory systems. This seems particularly likely in situations that involve few neuronal subtypes, such as the Drosophila retina, where four transcription factors or transcription factor combinations could act to stabilize the four color photoreceptor subtypes. In the mouse olfactory system that has over a thousand sensory neuron subtypes, a mechanism that involves a distinct transcription factor battery to maintain each identity seems unlikely. However, a generic transcription factor could act in each cell to maintain repression of all silent receptor genes.

\section{References}

Bargmann, C.I., Hartwieg, E., and Horvitz, H.R. 1993. Odorantselective genes and neurons mediate olfaction in C. elegans. Cell 74: 515-527.

Bauer Huang, S.L., Saheki, Y., VanHoven, M.K., Torayama, I., Ishihara, T., Katsura, I., van der Linden, A., Sengupta, P., and Bargmann, C.I. 2007. Left-right olfactory asymmetry results from antagonistic functions of voltage-activated calcium channels and the Raw repeat protein OLRN-1 in C. elegans. Neural Develop. 2: 24. doi:10.1186/1749-8104-2-24.

Chess, A., Buck, L., Dowling, M.M., Axel, R., and Ngai, J. 1992. Molecular biology of smell: Expression of the multigene family encoding putative odorant receptors. Cold Spring Harb. Symp. Quant. Biol. 57: 505-516.

Chuang, C.F. and Bargmann, C.I. 2005. A Toll-interleukin 1 repeat protein at the synapse specifies asymmetric odorant receptor expression via ASK1 MAPKKK signaling. Genes \& Dev. 19: 270-281.

Chuang, C.F., Vanhoven, M.K., Fetter, R.D., Verselis, V.K., and Bargmann, C.I. 2007. An innexin-dependent cell network establishes left-right neuronal asymmetry in C. elegans. Cell 129: 787-799.

Cook, T., Pichaud, F., Sonneville, R., Papatsenko, D., and Desplan, C. 2003. Distinction between color photoreceptor cell fates is controlled by Prospero in Drosophila. Dev. Cell 4: 853-864.

Fischbach, K.-F. and Dittrich, A.P. 1989. The optic lobe of Drosophila melanogaster. I. A Golgi analysis of wild-type structure. Cell Tissue Res. 258: 441-475.

Johnston Jr., R.J. and Desplan, C. 2008. Stochastic neuronal cell fate choices. Curr. Opin. Neurobiol. 18: 20-27.

Lans, H. and Hoeijmakers, J.H. 2006. Cell biology: Ageing nucleus gets out of shape. Nature 440: 32-34.

Lans, H., Rademakers, S., and Jansen, G. 2004. A network of stimulatory and inhibitory $\mathrm{G} \alpha$-subunits regulates olfaction in Caenorhabditis elegans. Genetics 167: 1677-1687.

Lesch, B.J., Gehrke, A.R., Bulyk, M.L., and Bargmann, C.I. 2009. Transcriptional regulation and stabilization of left-right neuronal identity in C. elegans. Genes \& Dev. 23: 345-358.

Lewcock, J.W. and Reed, R.R. 2004. A feedback mechanism regulates monoallelic odorant receptor expression. Proc. Natl. Acad. Sci. 101: 1069-1074.

Losick, R. and Desplan, C. 2008. Stochasticity and cell fate. Science 320: 65-68.

Maamar, H. and Dubnau, D. 2005. Bistability in the Bacillus subtilis K-state (competence) system requires a positive feedback loop. Mol. Microbiol. 56: 615-624.

Maamar, H., Raj, A., and Dubnau, D. 2007. Noise in gene expression determines cell fate in Bacillus subtilis. Science 317: 526-529. 
Malnic, B., Hirono, J., Sato, T., and Buck, L.B. 1999. Combinatorial receptor codes for odors. Cell 96: 713-723.

Mazzoni, E.O., Celik, A., Wernet, M.F., Vasiliauskas, D., Johnston, R.J., Cook, T.A., Pichaud, F., and Desplan, C. 2008. Iroquois complex genes induce co-expression of rhodopsins in Drosophila. PLoS Biol. 6: e97. doi:10.1371/journal. pbio.0060097.

Mikeladze-Dvali, T., Wernet, M.F., Pistillo, D., Mazzoni, E.O., Teleman, A.A., Chen, Y.W., Cohen, S., and Desplan, C. 2005. The growth regulators warts/lats and melted interact in a bistable loop to specify opposite fates in Drosophila R8 photoreceptors. Cell 122: 775-787.

Morante, J. and Desplan, C. 2004. Building a projection map for photoreceptor neurons in the Drosophila optic lobes. Semin. Cell Dev. Biol. 15: 137-143.

Peckol, E.L., Troemel, E.R., and Bargmann, C.I. 2001. Sensory experience and sensory activity regulate chemosensory receptor gene expression in Caenorhabditis elegans. Proc. Natl. Acad. Sci. 98: 11032-11038.

Sagasti, A., Hisamoto, N., Hyodo, J., Tanaka-Hino, M., Matsumoto, K., and Bargmann, C.I. 2001. The CaMKII UNC-43 activates the MAPKKK NSY-1 to execute a lateral signaling decision required for asymmetric olfactory neuron fates. Cell 105: 221-232.

Serizawa, S., Ishii, T., Nakatani, H., Tsuboi, A., Nagawa, F., Asano, M., Sudo, K., Sakagami, J., Sakano, H., Ijiri, T., et al. 2000. Mutually exclusive expression of odorant receptor transgenes. Nat. Neurosci. 3: 687-693.

Shykind, B.M., Rohani, S.C., O'Donnell, S., Nemes, A., Mendelsohn, M., Sun, Y., Axel, R., and Barnea, G. 2004. Gene switching and the stability of odorant receptor gene choice. Cell 117: 801-815.

Simpson, P. 1997. Notch signalling in development: On equivalence groups and asymmetric developmental potential. Curr. Opin. Genet. Dev. 7: 537-542.

Suel, G.M., Garcia-Ojalvo, J., Liberman, L.M., and Elowitz, M.B. 2006. An excitable gene regulatory circuit induces transient cellular differentiation. Nature 440: 545-550.

Sulston, J.E. and Horvitz, H.R. 1977. Post-embryonic cell lineages of the nematode, Caenorhabditis elegans. Dev. Biol. 56: $110-156$.

Tanaka-Hino, M., Sagasti, A., Hisamoto, N., Kawasaki, M., Nakano, S., Ninomiya-Tsuji, J., Bargmann, C.I., and Matsumoto, K. 2002. SEK-1 MAPKK mediates $\mathrm{Ca}^{2+}$ signaling to determine neuronal asymmetric development in Caenorhabditis elegans. EMBO Rep. 3: 56-62.

Troemel, E.R., Sagasti, A., and Bargmann, C.I. 1999. Lateral signaling mediated by axon contact and calcium entry regulates asymmetric odorant receptor expression in C. elegans. Cell 99: 387-398.

Vanhoven, M.K., Bauer Huang, S.L., Albin, S.D., and Bargmann, C.I. 2006. The claudin superfamily protein nsy-4 biases lateral signaling to generate left-right asymmetry in C. elegans olfactory neurons. Neuron 51: 291-302.

Wernet, M.F. and Desplan, C. 2004. Building a retinal mosaic: Cell-fate decision in the fly eye. Trends Cell Biol. 14: 576584.

Wernet, M.F., Mazzoni, E.O., Celik, A., Duncan, D.M., Duncan, I., and Desplan, C. 2006. Stochastic spineless expression creates the retinal mosaic for colour vision. Nature 440: 174-180. 


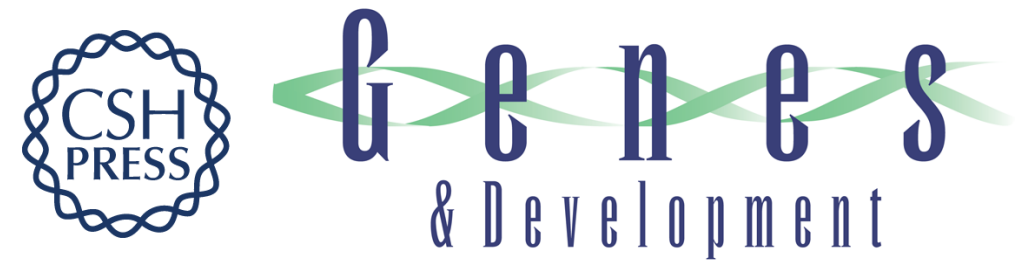

\section{Maintaining a stochastic neuronal cell fate decision}

Daniel Vasiliauskas, Robert Johnston and Claude Desplan

Genes Dev. 2009, 23:

Access the most recent version at doi:10.1101/gad.1780509

\section{Related Content Transcriptional regulation and stabilization of leftright neuronal identity in C. elegans \\ Bluma J. Lesch, Andrew R. Gehrke, Martha L. Bulyk, et al. \\ Genes Dev. February, 2009 23: 345-358 \\ References This article cites 31 articles, 9 of which can be accessed free at: \\ http://genesdev.cshlp.org/content/23/4/385.full.html\#ref-list-1 \\ Articles cited in: \\ http://genesdev.cshlp.org/content/23/4/385.full.htmI\#related-urls \\ License \\ Email Alerting Receive free email alerts when new articles cite this article - sign up in the box at the top Service right corner of the article or click here.}

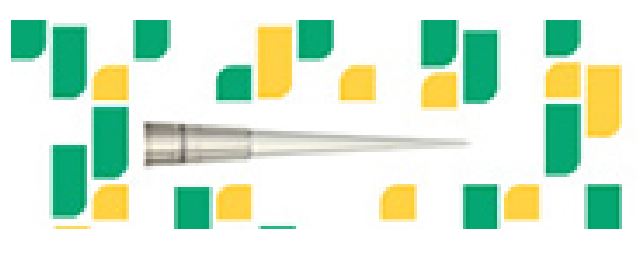

Focused on your science. 